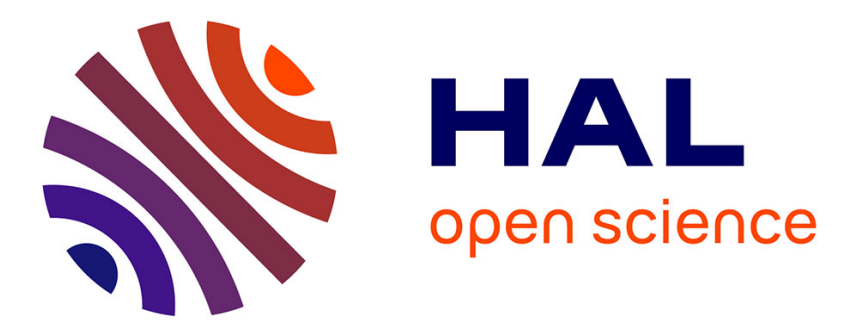

\title{
Implantable CMOS pixel sensor for positron imaging in rat brain
}

\author{
J. Heymes, L. Ammour, M. Bautista, G. Bertolone, A. Dorokhov, S. Fieux, F. \\ Gensolen, M. Goffe, C. Hu-Guo, M. Kachel, et al.
}

\section{- To cite this version:}

J. Heymes, L. Ammour, M. Bautista, G. Bertolone, A. Dorokhov, et al.. Implantable CMOS pixel sensor for positron imaging in rat brain. Nuclear Instruments and Methods in Physics Research Section A: Accelerators, Spectrometers, Detectors and Associated Equipment, 2018, 911, pp.19-24. 10.1016/j.nima.2018.09.117 . hal-01952201

\section{HAL Id: hal-01952201 https://hal.science/hal-01952201}

Submitted on 13 Mar 2019

HAL is a multi-disciplinary open access archive for the deposit and dissemination of scientific research documents, whether they are published or not. The documents may come from teaching and research institutions in France or abroad, or from public or private research centers.
L'archive ouverte pluridisciplinaire HAL, est destinée au dépôt et à la diffusion de documents scientifiques de niveau recherche, publiés ou non, émanant des établissements d'enseignement et de recherche français ou étrangers, des laboratoires publics ou privés. 


\title{
Implantable CMOS Pixel Sensor for Positron Imaging in Rat Brain
}

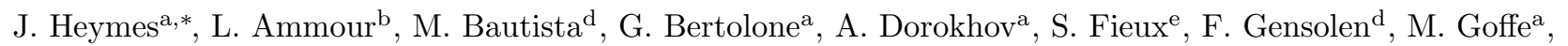

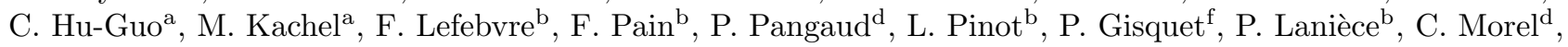 \\ M.-A. Verdier ${ }^{\mathrm{c}}$, M. Winter ${ }^{\mathrm{a}}$, L. Zimmer ${ }^{\mathrm{e}}$, J. Baudot ${ }^{\mathrm{a}}$ \\ ${ }^{a}$ Université de Strasbourg, CNRS, IPHC UMR 7178, F-67000 Strasbourg, France \\ ${ }^{b}$ IMNC UMR 8165, Université Paris-Sud, Université Paris Diderot, CNRS/IN2P3, Université Paris Saclay, 91405 Orsay, France \\ ${ }^{c}$ Univ. Paris-Diderot, Sorbonne Paris Cité, IMNC CNRS-IN2P3, 91405 Orsay, France \\ ${ }^{d}$ Aix Marseille Université, CNRS/IN2P3, CPPM, Marseille, France \\ ${ }^{e}$ CERMEP-Imagerie du vivant, Université Claude Bernard Lyon 1, CNRS, INSERM, Hospices Civils de Lyon, Lyon, France \\ ${ }^{\prime}$ NeuroPSI CNRS/INSB, Univ Paris Sud, Orsay, France
}

\begin{abstract}
IMIC is a Monolithic Active Pixel Sensor prototype designed for the MAPSSIC project, which aims at developing wireless intracerebral probes dedicated to image positron-emitting source activity in the brain of awake and freely moving rats. Former experiments with the PIXSIC positron probe based on a passive sensor have validated the proof of concept, but have also shown limitations with regards to the probe robustness and to its transparency to annihilation photons.

The IMIC circuit features a matrix of $16 \times 128$ active pixels of $30 \times 50 \mathrm{\mu m}^{2}$ size and targets to overcome the PIXSIC probe drawbacks by exploiting a thin sensitive layer of $18 \mu \mathrm{m}$, still featuring an overall thickness close to $300 \mu \mathrm{m}$. Additionally, by using a low power $(55 \mathrm{nW} /$ pixel) in-pixel front-end architecture providing binary output, IMIC solves the challenge of implanting an active sensor in tissues where overheating is forbidden.

The needle-shaped sensor $610 \mu \mathrm{m} \times 12000 \mu \mathrm{m}$ was fabricated and tested in laboratory. The whole sensor dissipates $160 \mathrm{\mu W}$ and its imaging capabilities were asserted with various sources : ${ }^{55} \mathrm{Fe},{ }^{90} \mathrm{Sr}$ and ${ }^{18} \mathrm{~F}$. These tests also demonstrated robust count-rate measurement with IMIC in the range 10-1000 counts/matrix/s. Finally, a dedicated setup qualitatively confirmed excellent in-sensitivity to $511 \mathrm{keV} \gamma$-rays.

In this paper, we present the sensor requirements and its detailed design. We also discuss the first characterisation results and the outlook for the integration of IMIC into an implantable probe.
\end{abstract}

Keywords: Semiconductor Detectors, CMOS Pixel Sensors, Beta Probe, Brain Imaging, Neuroimaging

\section{Introduction}

Neuroimaging results of anaesthetised or highly restrainęid animals suffers from undesired effects that are limiting ${ }_{22}$ their validity $[1,2]$. In contrast, real time monitoring of ${ }_{23}$ molecular processes in brains of fully awake animals has a ${ }_{24}$ strong potential to improve the robustness of observations ${ }_{25}$ that couple neuroimaging and behavioural studies $[3,4]{ }_{26}$ Approaches based on PET imaging $[5,6,7]$ have only ${ }_{27}$ partly achieved this goal due to a remaining mechanical ${ }_{28}^{27}$ support, or an overall weight, or a limited space that does ${ }_{29}$ not guarantee the animal freedom of movement for behavioral studies.

Another approach consists in performing invasive measurements using an autonomous positron sensitive probe ${ }_{34}$ implanted in specific brain regions by stereotaxic surgery. ${ }_{35}$ An advanced solution using a needle-shaped passive silicon ${ }_{36}$ sensor was developed with PIXSIC [8, 9, 10, 11]. Figure $1_{37}$

*. Corresponding author

Email address: julian.heymes@iphc.cnrs.fr (J. Heymes) allows wireless data transmission through a relative light system carried on a backpack. PIXSIC actually releases physical hindrances to the rodent movements. While invasiveness of this technique is a major point of concern, no impact from the small-size probe direct implantation was observed. However, the PIXSIC setup suffers from two main limitations.

First the length of the metallic lines that connect pixel sensors to their pre-amplification stages on the PICPUS readout chip induces EM pickup, which reduces signal over noise ratio and hence the overall probe sensitivity.

The second weak point of PIXSIC pertains to the thickness of the passive sensor, which is constrained by antagonist requirements. On the one hand, mitigating the sensitivity to $\gamma$-rays generated by electron-positron annihilations, whose positions are not necessarily near the probe, requires a thickness as small as possible. On the other hand, a relatively thick sensor is beneficial to mechanical stiffness. A compromise of $200 \mu \mathrm{m}$ thickness was chosen for PIXSIC, though 


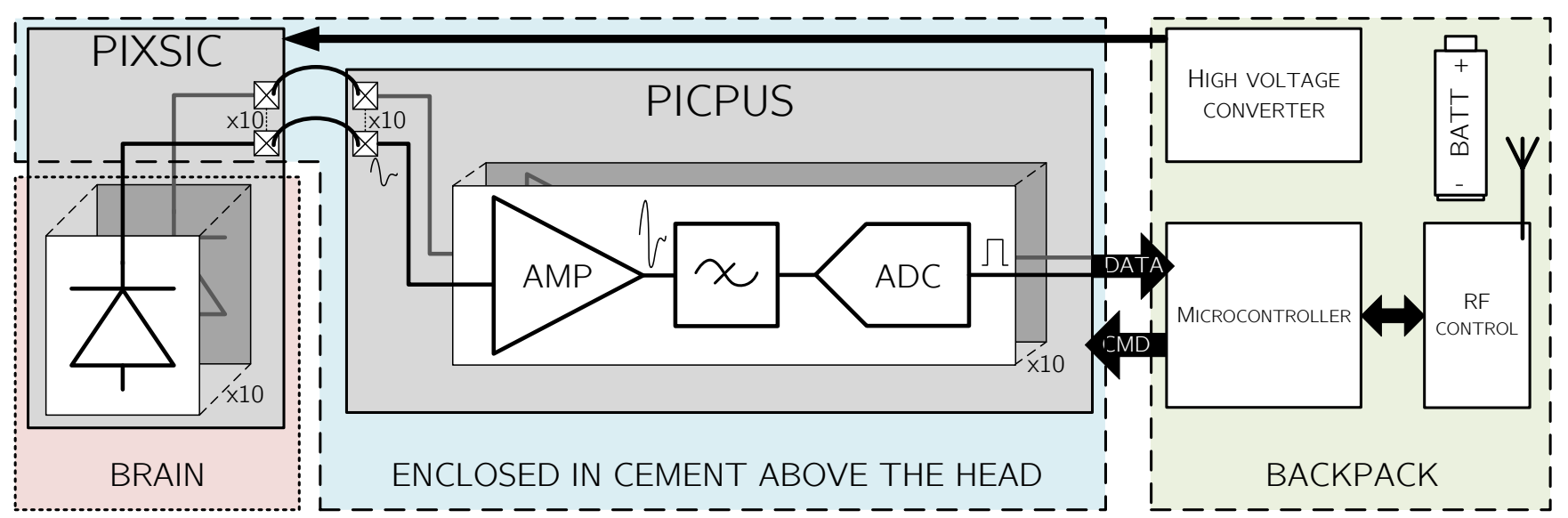

FIGURE 1: Schematic of the PIXSIC setup. The needle probe implanted in the brain is composed of 10 passive pixels, each directly wire-bonded to one channel of the readout chip : PICPUS. This chip embeds amplifiers, filter, digitisation, and memories, and is enclosed in dental cement above the skull. A micro-controller steers and gathers data from the readout chip. It is located on a backpack carried by the rodent during experiments. It also embeds active and passive circuits for the polarisation of the sensor and readout chip, and a battery for full autonomy.

it was not really satisfactory for both the constraints.

The usage of monolithic active pixels sensors (MAPS) 78 was proposed [12] to improve the implanted probe concept. 79 MAPS can embed an amplification and digitisation stage in each pixel, basically replacing the PICPUS chip and suppressing a priori sensitivity to electromagnetic envi- ${ }^{80}$ ronment. Since the amplification allows a small primary ${ }_{81}$ signal, the sensitive thickness can be thinner than the overall sensor thickness. Hence a depth of typically a few tens ${ }_{83}$ of micrometers generate enough signal for efficient detection and does not need to extent to the full sensor thickness also limiting its sensitivity to $\gamma$-rays. The latter can ${ }_{86}$ be set optimally with respect to invasiveness and stiffness. ${ }_{87}$ In addition, the probe integration is simplified due to the ${ }_{88}$ absence of an additional circuit on the rodent head, as de- ${ }_{89}$ picted in figure 2.

However, the usage of an implanted active sensor can only be effective under the condition that the local tempe- ${ }_{93}$ rature is marginally affected by the probe operation (typi- ${ }_{94}$ cally $\Delta T<1^{\circ} \mathrm{C}$ ). Consequently, a key design feature is the ${ }_{95}$ power dissipated by the sensor.

Following the ideas developed in [12] along with the requirement to minimize the power consumption, a first ${ }_{99}$ MAPS based on the CMOS pixel sensors technology has ${ }_{100}$ been developed. The chip is named IMIC (Imageur Moléculquire Intra-Cérébral) and is part of the MAPSSIC (MAPS Sonde ${ }_{102}$ Intra-Crânienne) project to develop and exploit the first ${ }_{103}$ MAPS-based intracerebral positron probe.

This paper first describes in section 2 the for the active sensor to be implanted in the brain. The design and operation features of IMIC, optimized for $\operatorname{low}_{108}$ count rate and power dissipation, are detailed in section 3. Section 4 provides first characterization results, focussing on functional validation and sensitivity with laboratory sources.

\section{Sensor Requirements}

Neuroimaging exploits a variety of radioactive isotopes, typically ${ }^{18} \mathrm{~F},{ }^{15} \mathrm{O}$ and ${ }^{11} \mathrm{O}$, which emit $\beta+$ at energies from 0 to $1.7 \mathrm{MeV}$. The probe ability to measure activities depends obviously on what energy range the sensor is sensitive to. Consequently an important figure of merit is the detection threshold, which should be as low as possible (this requirement is an outcome of the study in [12]) and depends on the signal-over-noise ratio of the sensing element. The threshold value will be discussed in the next section 3 in view of the sensitive thickness and noise level of the designed sensors.

As previously mentionned, the usage of MAPS for this kind of probe intends to both enhance the overall robustness and simplify the system beyond the implanted part of the probe. Thus, we have included into the requirements the digital conversion of the signal inside the pixels hence facilitating further data transmission. It is also expected that the readout architecture of the sensor optimises data throughput to the micro-controller embedded on the animal backpack with respect to the measured activity.

Regarding the sensors dimensions, we follow the ones of the PIXSIC sensor ( $15 \mathrm{~mm}$ long, $500 \mu \mathrm{m}$ wide and $500 \mu \mathrm{m}$ thick), compatible with the requirements on invasiveness [9].

Imaging the source and evaluating its activity require some level of segmentation, especially along the (vertical) implantation direction. The size of the pixels of the PIXSIC 


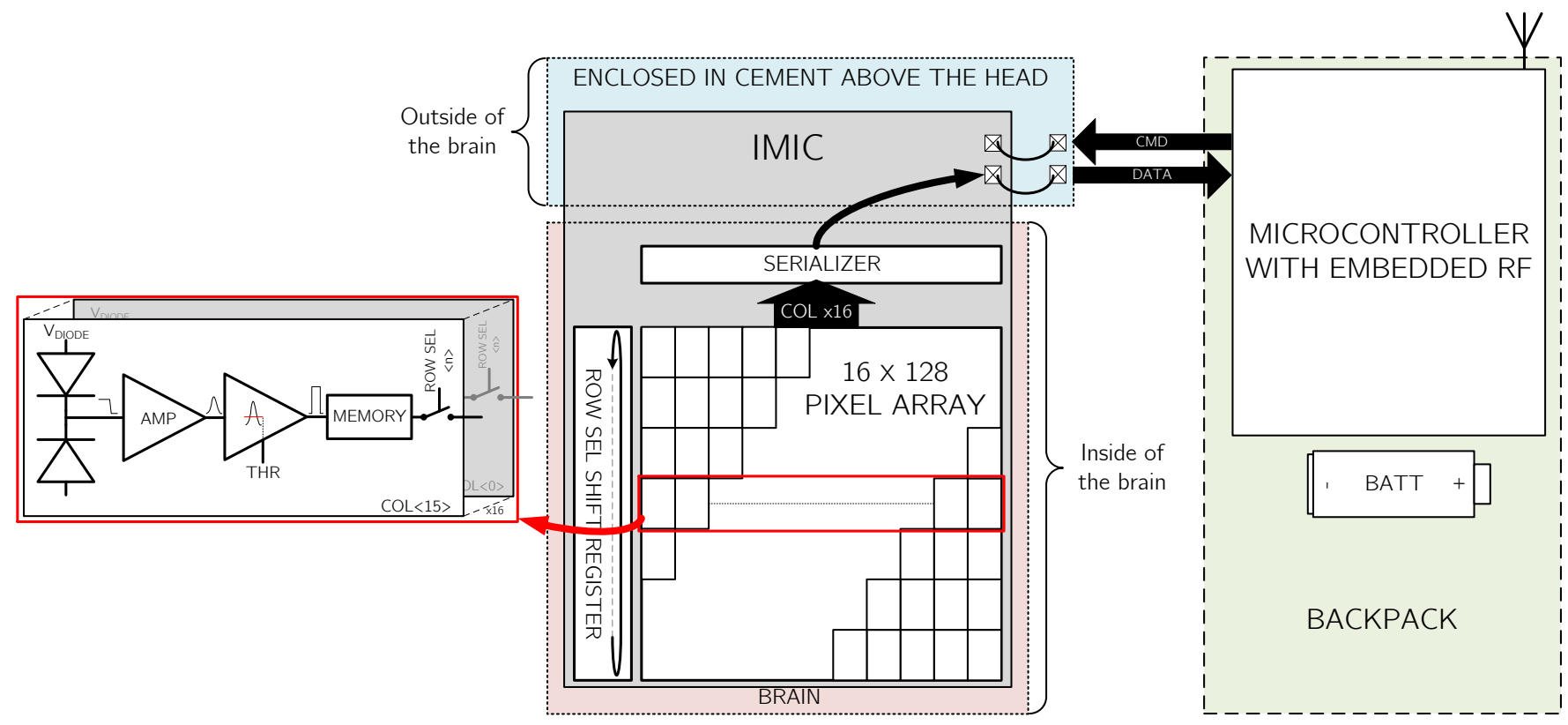

Figure 2: Schematic of the MAPSSIC setup. The monolithic active pixel sensor IMIC is a needle shaped probe composed of $16 \times 128$ pixels of $30 \times 50 \mathrm{\mu m}^{2}$, which provide binary information whether a pixel has been hit or not. IMIC is wire bonded to a PCB acting as an interface to the connector and is enclosed in cement on the head of the rodent. The chip is steered and read out only through digital signals by using a micro-controller located on the backpack together with a battery, which is embedding wireless transmission with the data acquisition PC.

probe were $200 \times 500 \mathrm{\mu m}^{2}$, with no segmentations but139 along its length. Such a segmentation proved to be granu-140 lar enough to map subcortical brain activity [9] and there-141 fore should be matched quite easily by MAPS, which can ${ }_{142}$ feature much higher granularity.

According to first positron count rate observations with ${ }^{143}$ the PIXSIC probe [9], an average positron flux of about ${ }_{144}$ $100 \mathrm{hits} /$ sensor $/ \mathrm{s}$ is expected over the required dimensions. This level of positron flux corresponds to at least three orders of magnitude less than standard achievements of ${ }^{146}$ CMOS pixel sensors used for charge particle detection. ${ }^{14}$ However, a stringent constraint arises from operation in ${ }^{148}$ a living animal brain.

As a matter of fact, active architectures involve power ${ }^{15}$ consumption and thus heat dissipation, which could locally ${ }^{152}$ overheat brain tissues in contact with the sensor. To avoid ${ }^{153}$ behavioural impact or worst, tissue damage, from excess ${ }^{154}$ heat dissipation, finite element computations were perfor- ${ }^{155}$ med in order to set a limit on the dissipated power. The si- ${ }^{156}$ mulated geometry includes a volume of silicon representing ${ }^{157}$ the implanted part of the probe $(10 \mathrm{~mm} \times 500 \mu \mathrm{m} \times 600 \mu \mathrm{m})^{158}$ in a spheric medium representing the brain at a constant ${ }^{159}$ temperature of $37^{\circ} \mathrm{C}$. The highest temperature gradient ${ }^{160}$ at the direct probe contact stays below the required $1{ }^{\circ} \mathrm{C}^{161}$ for a dissipated power below $4 \mathrm{~mW}$. Considering the pos- ${ }^{162}$ sible implantation of multiple probes composed of 2 to $4^{163}$ sensors, we have set a limit of $1 \mathrm{~mW}$ dissipated per sensor. ${ }^{164}$ Due to the simplistic nature of the above calculation, the ${ }^{165}$ power of $1 \mathrm{~mW}$ per sensor is taken as an indicative limit. The actual guideline chosen for the sensor design follows the requirement to achieve the lowest possible power dissipation.

\section{IMIC sensor design}

\subsection{Technology choice and overall geometry}

We have chosen to design the sensor in the same imager CMOS process with $180 \mathrm{~nm}$ feature size as used for various charged particle detection applications [13]. The sensitive volume consist of a $18 \mu \mathrm{m}$ thick low-doped epitaxial layer, which has demonstrated $100 \%$ detection efficiency for minimum ionising particles for various pixel sizes below $50 \mu \mathrm{m}[13]$. Such realization guarantee a priori excellent detection performances for $\beta+$ within the energy range of interest.

In addition, the thin detection layer presents two key advantages. First, it mitigates the sensitivity to the annihilation photons as recommended in [12]. Secondly, it allows thinning the sensor down to thicknesses from 100 to $300 \mu \mathrm{m}$, thus achieving an optimum between invasiveness and mechanical stiffness.

Regarding segmentation, we have opted for a rectangular shape pixel, within the dimensions known to yield excellent charge collection : $30 \mu \mathrm{m}$ wide and $50 \mu \mathrm{m}$ along the vertical dimension of the probe. A matrix of 16 (horizontal $) \times 128$ (vertical) pixels then covers a sensitive area of $480 \times 6400 \mathrm{\mu m}^{2}$, meeting the objective to create a 


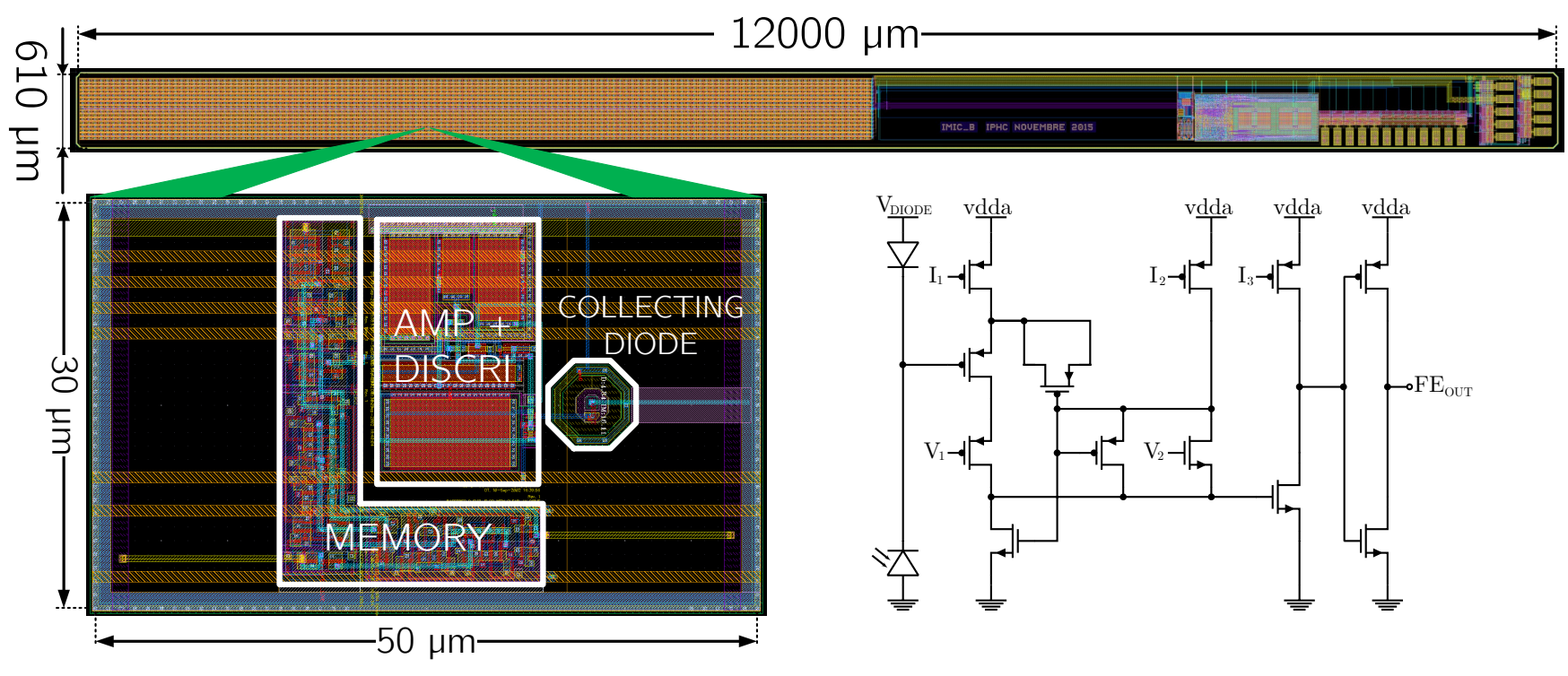

Figure 3: Top : layout of the $12000 \mu \mathrm{m}$ long and $610 \mu \mathrm{m}$ wide IMIC sensor. Bottom left : magnified layout of a single pixel underlying the location of its main functional components. Bottom right : Pixel circuit schematic derived from the ALPIDE sensor [14].

needle-shape probe.

The overall sensor dimensions reach $610 \mu \mathrm{m}$ width and ${ }_{201}$ $12 \mathrm{~mm}$ length. The vertical extension beyond the active 202 matrix serves to implement the readout and control micro-203 circuits as well as the connection pads. The sensor is thin-204 ned by the foundry to $280 \mu \mathrm{m}$ and hence presents three 205 different layers along its thickness : first $10 \mu \mathrm{m}$ of elec-206 tronic process insensitive to particles, secondly $18 \mu \mathrm{m}$ of 207 sensitive epitaxy and lastly roughly $250 \mu \mathrm{m}$ of silicon sub-208 strate that provides mechanical support.

The produced sensor layout is shown on the top of fi-211 gure 3 .

\subsection{Pixel design}

To satisfy simultaneously the main requirements on the ${ }^{215}$ pixel functionalities and performances (digital output, low data-throughput, low detection threshold and low power), ${ }^{216}$ a modified version of the ALPIDE (ALice PIxel DEtec-217 tor) [14] circuit developed for the ALICE experiment is 218 implemented. The initial compact pixel architecture am-219 plifies, shapes (with clipping to prevent saturation), and ${ }_{220}$ discriminates the signal collected by the diode using only221 12 transistors, as depicted on the bottom of figure 3). ${ }_{222}$ Pixels integrate any incoming charge between two conse-223 cutive readouts. Since the binary output of the front-end 224 indicating the presence of a hit is only available for a few 225 tens of $\mu \mathrm{s}$, a 1-bit memory was added per pixel to keep this226 information until the pixel readout time. The memory is 227 reset after the pixel is read out, so that if a second particle hit the same pixel before readout, the 1-bit memory can-228 not account for it. The matrix readout strategy is detailed in the next sub-section.

Simulations of the pixel architecture using Cadence Virtuoso Analog Design Environment have evaluated the equivalent noise charge (ENC) at the collection node below 10 electrons, hence allowing to set discriminators for the digitisation at an equivalent level of $\approx 100 \mathrm{e}^{-}$. The corresponding energy threshold for the detection equals $\approx 360 \mathrm{eV}$, well below the maximum probability value of energy loss in $18 \mu \mathrm{m}$ of silicon by $\beta+$ with energies within 0 to $2 \mathrm{MeV}$.

Finally, these simulations predict that the overall frontend dissipates about $55 \mathrm{nW}$ per pixel (or $113 \mu \mathrm{W}$ for the whole matrix), well below the upper limit. The schematic of the pixel and its layout are shown in figure 3 .

\subsection{Readout strategy}

IMIC readout mechanism is kept simple for the sake of space and power dissipation, which does not depend on the hit rate, and relies on the rolling-shutter principle. A token is propagated through the matrix in a shift register at a fixed clock rate to select one line. The 16-bits word formed by all the pixel memories in the line is serialised towards the output of the sensor at a higher clock rate (at least 16 times), which defines $f_{\text {clock }}$. This operation is repeated 128 times to gather data from the entire pixel array, thus the time $t_{\text {readout }}$ needed for this full readout cycle amounts to

$$
t_{\text {readout }}=\frac{2048}{f_{\text {clock }}} \quad[\mathrm{s}] .
$$




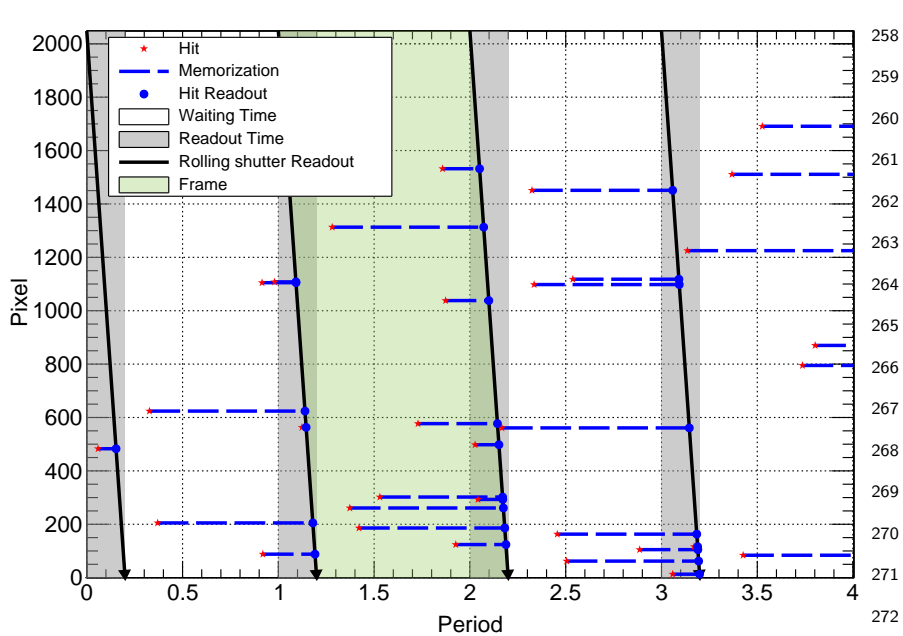

Figure 4: Readout strategy for IMIC. When a pixel detects a hit, the event is memorised until rolling-shutter readout. A frame is composed of an adjustable waiting time and the readout time. During the readout, the data of each pixel is sent outside the sensor, bit after bit. The data of the memory is reset after readout to allow the detection and memorisation of a new hit.
Pixels integrate particle hits during the time $t_{\text {wait }}$ between two readout cycles, plus the readout time itself :

$$
t_{\text {integration }}=t_{\text {readout }}+t_{\text {wait }} \quad[\mathrm{s}] .
$$

For each readout cycle, data are provided to the microcontroller as a vector of 2048 bits to be sent further away through a wireless protocol. In view of limiting the data273 rate defined by equation 3 and hence the overall power consumption of the MAPSSIC setup, it is better to maximise the waiting time $t_{\text {wait }}$ during two readout cycles. However, avoiding pile-up of hits during the same integration period requires to minimise $t_{\text {wait }}$.

$$
\text { Data rate }=\frac{2048}{t_{\text {integration }}} \quad[\mathrm{bits} / \mathrm{s}]
$$

The foreseen average hit-rate of about 100 hits/matrix/s in our experiments, leads to an optimisation for the integration time of around 50 millisecond. In such conditions the data rate stays at a few tens of kilo-bits per second while keeping the pile-up probability at the per mil level. Figure 4 depicts the principle of operation of the IMIC readout.

\subsection{A fully programmable needle shaped sensor}

In order to minimize the complexity of the external circuitry, IMIC limits the number of required connections by using a command decoder controlled through Serial Peripheral Interface (SPI) protocol. This controller also allows the parametrisation of on-chip digital-to-analog converters (DACs) used for the polarisation of the front-end. The steering signals (and the data) are sent independently bet-275 ween the micro-controller located on the rodent backpack ${ }^{276}$ and the sensor. The micro-controller connects to the external world through a wireless protocol. The overall system description will be the subject of a subsequent publication.

The digital block driving IMIC is located on the sensor edge near the few connection pads located outside of the brain, opposite to the pixel matrix deeply implanted in the tissues. This block adds a power consumption of approximately $2 \mu \mathrm{W}$. The simulated total IMIC power consumption amounts to approximately $115 \mu \mathrm{W}$. This dissipation does not depend on the activity measured but is entirely driven by the number of pixels populating the matrix.

The main characteristics of the IMIC sensor are summarised in table 1.

TABLE 1: Summary of the simulated characteristics of the IMIC sensor.

\begin{tabular}{ll}
\hline \hline Feature & Value \\
\hline Sensor size $\left[\mathrm{\mu m}^{3}\right]$ & $610 \times 12000 \times 280$ \\
Pixel pitch $\left[\mathrm{\mu m}^{2}\right]$ & $30 \times 50$ \\
Sensitive depth $[\mu \mathrm{m}]$ & 18 \\
Det. threshold $[\mathrm{eV}$ deposited] & $\approx 400$ \\
Pixel power consumption $[\mathrm{nW} / \mathrm{pixel}]$ & $\approx 55$ \\
Matrix size & $16 \times 128$ \\
Sensitive area $\left[\mathrm{\mu m}^{2}\right]$ & $480 \times 6400$ \\
Total power consumption $[\mu \mathrm{HW}]$ & $\approx 115$ \\
\hline \hline
\end{tabular}

\section{IMIC characterisation}

\subsection{First images}

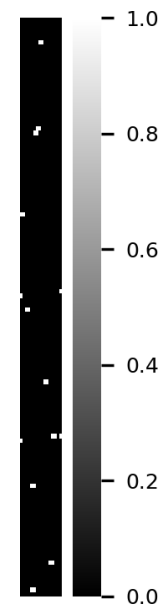

(a)

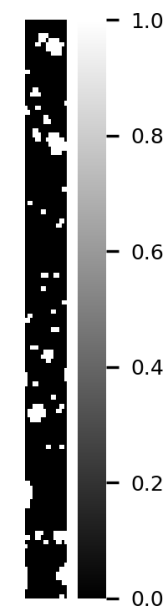

(b) ${ }^{90} \mathrm{Sr}$

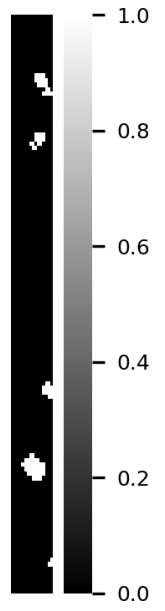

(c)

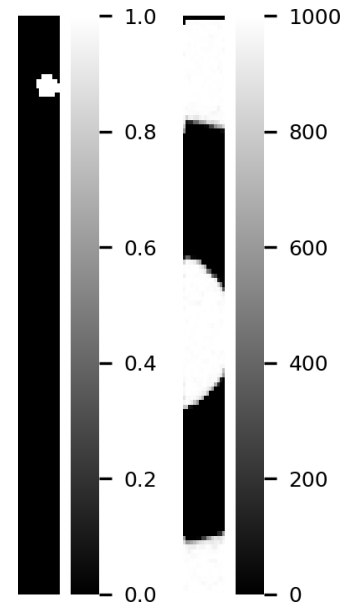

(d)

(e)
FiguRE 5: Images acquired with the IMIC sensor. Single frame aquired with a ${ }^{55} \mathrm{Fe}$ soft-X-ray source (5a), a ${ }^{90} \mathrm{Sr} \beta$ source $(5 \mathrm{~b})$, and a ${ }^{18} \mathrm{~F}$ positron source $(5 \mathrm{c})$. A single annihilation photon from the ${ }^{18} \mathrm{~F}$ positron source hit $(5 \mathrm{~d})$. Image composed of 1000 accumulated frames of a metallic spacer acquired with ${ }^{55} \mathrm{Fe}(5 \mathrm{e})$.

A few IMIC sensors were bonded to a printed circuit board (PCB) in order to facilitate the first tests. After adjustment of the various polarisations through the on-chip 
DACs driven by the SPI protocol, the bonded chips were 333 confirmed to be fully functional. All results reported below 334 were obtained with these sensors at room temperature and 335 under relative obscurity.

The measured dissipated power by the whole sensor is $160 \mu \mathrm{W}$, close to the value expected from simulations in table 1 considering the low absolute value for a complete chip.

Three types of sources have been used to characterise the IMIC sensor. Single frames from each source type are displayed in figures $5 \mathrm{a}-5 \mathrm{~d}$. Soft X-rays $(5.9$ and $6.4 \mathrm{keV}$ rays) from the ${ }^{55} \mathrm{Fe}$ source (figure $5 \mathrm{a}$ ) generate small clusters of mainly two pixels, while the $\beta$ particles, either from $\beta$ - $\left({ }^{90} \mathrm{Sr}\right.$ : figure $\left.5 \mathrm{~b}\right)$ or from $\beta+\left({ }^{18} \mathrm{~F}\right.$ : figure $\left.5 \mathrm{c}\right)$ yield clusters about ten times larger in average. This difference comes from the relatively long ionising path of $\beta$-rays in silicon. In contrast, X-rays undergo point-like conversion through photoelectric effect.

Images from $511 \mathrm{keV} \gamma$-rays were obtained by placing the ${ }^{18} \mathrm{~F}$ source far away (see sub-section 4.3 ) from the sensor. The cluster size observed for these $\gamma$-rays is similar to the one for positrons (see figure $5 \mathrm{~d}$ ). This indicates that the cluster size information cannot be used to identify (and suppress) the unwanted $\gamma$-rays hits on an individual basis information.

To demonstrate that the sensor internal readout per- ${ }^{33}$ forms as expected, an image of a metallic washer was ac- ${ }^{339}$ quired using the ${ }^{55} \mathrm{Fe}$ source (as shown in figure 5e). The ${ }^{340}$ ring is approximately $500 \mathrm{\mu m}$ thick with an external dia- ${ }^{341}$ meter of $5 \mathrm{~mm}$ and an opening diameter of $2 \mathrm{~mm}$. The ring structure can easily be recognized on the image as it ${ }^{343}$ completely stops the soft X-rays.

\subsection{Validation of long integration times}

As explained in section 3 , the modest awaited activities ${ }^{347}$ of the radiotracers allow running the IMIC sensor with $\mathrm{a}^{348}$ long integration time in the order of $100 \mathrm{~ms}$, which in turn $^{349}$ reduces the data flow and simplifies the whole system. This ${ }^{350}$ readout strategy is made possible by the in-pixel memory ${ }^{351}$ and was tested by measuring a range of activities at inte- ${ }^{-352}$ gration times varying from $20 \mathrm{~ms}$ to $1 \mathrm{~s}$. For this test, the IMIC sensor was indirectly illuminated ${ }^{354}$ with a ${ }^{90} \mathrm{Sr} \beta$ source through an aluminium shield. The ${ }^{355}$ activity was modulated using three different aluminium ${ }^{356}$ thicknesses : 1,3 and $7 \mathrm{~mm}$. Additional measurements ${ }^{357}$ were performed without the source in order to monitor ${ }^{358}$ dark count rate. For each combinations of shield and integration time, 1000 frames were acquired with the same ${ }^{359}$ detection threshold. Results are presented in figure 6 . The 360 mean entries per integration time were converted into a 361 mean activity measured over the whole matrix :

$$
\overline{\mathcal{A}\left(t_{\text {int }}\right)}=\frac{\overline{\text { entries }}}{t_{\text {int }}} \quad[\text { hits } / \text { matrix } / \mathrm{s}] \text {. }
$$

The saturation limit corresponds to the situation where every pixel over the matrix has been fired. This limit depends on the integration time through the simple relation :

$$
\mathcal{A}_{\text {sat }}\left(t_{\text {int }}\right)=\frac{2048}{t_{\text {int }}} \quad[\text { hits } / \text { matrix } / \mathrm{s}] \text {. }
$$

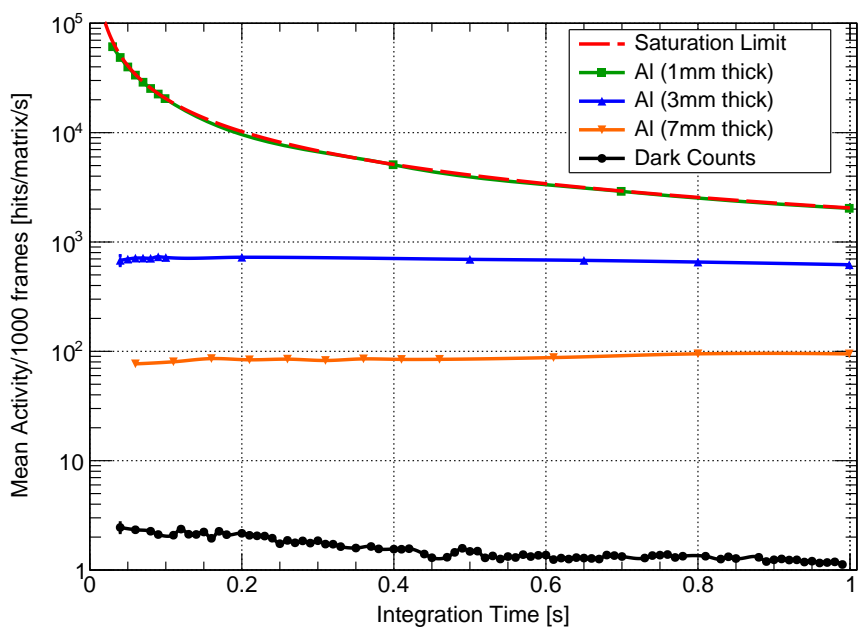

FiguRE 6: Mean activity seen by the sensor for integration times between $20 \mathrm{~ms}$ and $1 \mathrm{~s}$ of the IMIC sensor dark counts, and with various activities of ${ }^{90} \mathrm{Sr} \beta$-rays modulated by using various aluminum shielding thicknesses.

The analysis of the dark count map revealed that a few pixels (denominated noisy pixels in the following) dominate the production of fake hits, which explains the dark count rate evolution with the integration time. As there are one to two noisy pixels systematically firing during one integration period, it follows that the dark count rate simply decreases as $1 / t_{\text {integration }}$ along with equation 4 . The low level for the dark count rate means that the IMIC sensor can measure robustly activities down to approximately $10 \mathrm{hits} /$ matrix /s.

With the ${ }^{90} \mathrm{Sr}$ source attenuated by only $1 \mathrm{~mm}$ of aluminum, the mean activity follows the saturation limit curve. At $t_{\text {int }}=10 \mathrm{~ms}$, the mean activity seen by the sensor is approximately 60000 hits /matrix/s.

Using thicker shielding, the mean activity on the sensor remains below the saturation limit and is constant for every integration time. Thus, in the ballpark of expected average activities (100 hits/matrix/s), integration time up to $1 \mathrm{~s}$ can be chosen without information loss and with a safety margin against activity fluctuation of one order of magnitude. This read-out condition allows low data rates, and limits the power consumption of the overall system.

\subsection{Preliminary sensitivity study}

Measurements using ${ }^{18} \mathrm{~F}$ radioisotope, produced at the CYRCé cyclotron at IPHC, in a non-sealed aqueous solution were performed in view of studying the differential sensitivity to prompt $\beta+$ and annihilation photons. 


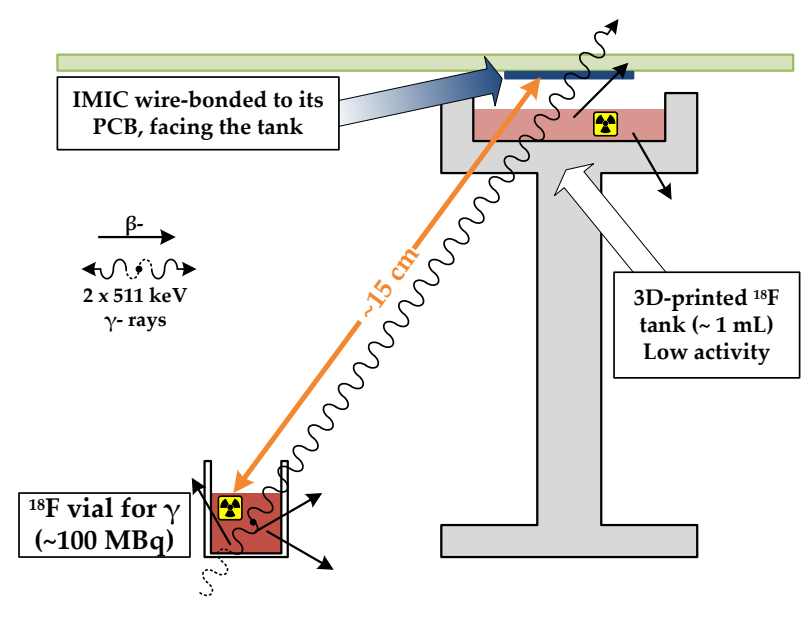

(a)

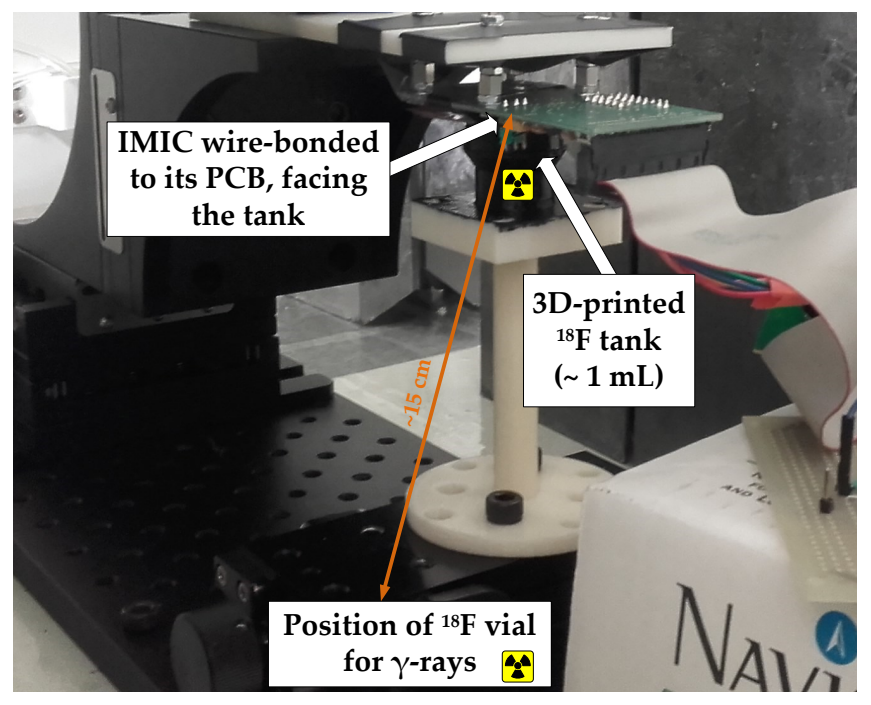

(b)

Figure 7: Experimental setup dedicated to measurements with ${ }^{18} \mathrm{~F}$. The IMIC sensor wire-bonded to its PCB is closely oriented towards a first tank containing an aqueous solution of ${ }^{18} \mathrm{~F}$. A vial of the same radiotracers with an higher activity was put on demand $15 \mathrm{~cm}$ away from the sensor in order to mimic the presence of a parasitic $\gamma$-ray source.

presenting an activity of about $1 \mathrm{MBq}$. The tank was de-376 signed to precisely position the sensor bonded to its $\mathrm{PCB}_{377}$ $5 \mathrm{~mm}$ away from the solution. Since the source was dissol-378 ved in saline solution, the sensor was protected by plastic 379 wrap.

Another vial filled with the same isotope but featuring азв much higher activity of $100 \mathrm{MBq}$ was disposed on demand 382 $15 \mathrm{~cm}$ away from the sensor. This second tank served as азвз parasitic source of $511 \mathrm{keV} \gamma$-rays, since the air distance 384 and the glass of the vial (the opening needed to fill the 385 vial is not oriented towards the sensor), fully attenuates386 positrons. This setup presented in figure 7 intends to reproduce roughly the head-bladder relative positions in a rat.

Data were taken with the IMIC sensor in three configurations, for 5 minutes each. The observed counts per time and average count rate are plotted in figure $8 \mathrm{a}$, where the average count rates are corrected for the time decay of ${ }^{18} \mathrm{~F}$. Count distributions observed with both tanks present are labeled ${ }^{18} \mathrm{~F}+\gamma$, the distributions obtained with only the tank close to the sensor is tagged ${ }^{18} \mathrm{~F}$, and finally the label

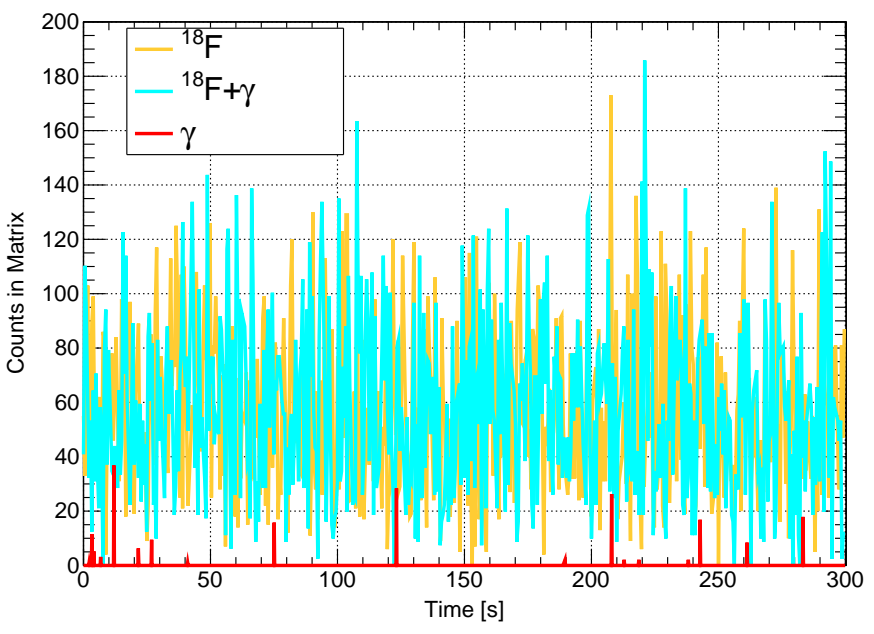

(a)

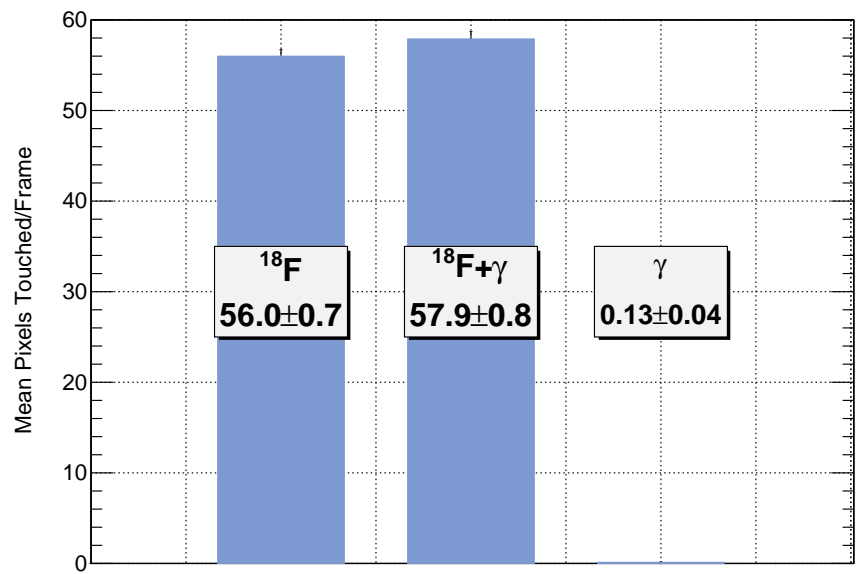

(b)

Figure 8: Left : Evolution with time of the pixel count per $100 \mathrm{~ms}$ frame duration for the three source configurations described in the text. Right : Average pixel count per frame over five minutes data acquisition for the same configurations. 
is $\gamma$ when only the distant tank was used.

The counting rates for the three source configurations 44 are displayed in figure $8 \mathrm{~b}$. Within uncertainties, the ave-442 rage counting rate with both sources $\left({ }^{18} \mathrm{~F}+\gamma\right)$ or only with ${ }_{443}$ the closer tank $\left({ }^{18} \mathrm{~F}\right)$ are equivalent, they amount respec-444 tively to $56.0 \pm 0.7$ and $57.9 \pm 0.8$ pixels/frame. This observation is coherent with the very small counting rate $(0.13 \pm 0.04$ pixels/frame $)$ measured when only the far- ${ }^{445}$ away source $(\gamma)$ is used.

The experiment illustrates qualitatively the very small re-447 lative sensitivity of the thin IMIC sensor to $\gamma$-rays with ${ }_{449}^{448}$ respect to $\beta+$.

\section{Conclusion}

Following [12], we have designed a monolithic active455 pixel sensor to equip the next generation of intracerebral ${ }^{456}$ positron probes for deep brain imaging adapted to fully ${ }_{458}^{457}$ conscious and freely moving rodents. The IMIC chip fea-559 tures 2048 active pixels over a sensitive area of $480 \times 64004 \mathrm{pm}^{2}$ The in-pixel amplification and 1-hit binary memory allows ${ }^{461}$ both a low detection threshold (400 eV for the energy de- ${ }_{463}^{462}$ posited) and fully digital matrix readout. This front-end ${ }_{464}$ architecture benefits from an extremely low power, addres-465 sing the main challenge of heat dissipation within the $\mathrm{im}^{-{ }^{466}}$ plantation tissues. Finally, being fabricated on a $18 \mu \mathrm{m}_{468}^{467}$ thick sensitive layer, the sensor is mostly transparent to469 $\gamma$-rays.

The first IMIC sample characterisations confirmed the ${ }^{471}$ successful operation of the sensor as a $\beta+$ activity loca- ${ }^{472}$ tor. The measured power dissipation per sensor reached ${ }_{474}$ $160 \mu \mathrm{W}$. This performance sets the current state-of-the- ${ }^{475}$ art for a probe built on the CMOS pixel technology, $\operatorname{and}_{477}^{476}$ should allow the implantation of a few probes in a single 478 animal with acceptable overheating of the local brain tis-479 sues. Foreseen in-vivo experiments will be conducted to $\mathrm{o}^{480}$ investigate the full compliance of the IMIC sensor with ${ }_{482}^{481}$ the application requirements.

A second generation sensor can benefit from a fully ${ }^{485}$ depleted thin sensitive layer, which would allow to increase ${ }^{486}$ the pixel size, hence decrease the total number of pixels ${ }_{488}$ and leading to a reduction of the total dissipated power. ${ }^{489}$

Currently, pairs of IMIC sensors are assembled back-to- ${ }^{490}$ back to form the implantable part of the final MAPSSIC ${ }_{492}^{491}$ probe with a total volume of $560 \times 610 \times 12000 \mathrm{\mu m}^{3}$. The ${ }_{493}$ rest of the construction process for the whole probe and ${ }^{494}$ its tests in realistic environment will be the subject of $\mathrm{a}^{495}$ forthcoming publication.

\section{Acknowledgement}

This work was partly funded in 2015 and 2017 by the 502 project Instrumentation aux limites from CNRS. This work was partly funded by France Life Imaging, $\quad{ }_{504}$
FLI/ANR-11-INBS-0006, of the French Investissements d'Avenir program run by the Agence Nationale pour la Recherche.

This work was partly funded by the IN2P3.

The authors thank the CYRCé team at IPHC for producing and making available the ${ }^{18} \mathrm{~F}$ solutions.

\section{References}

[1] Y.-R. Gao, Y. Ma, Q. Zhang, A. T. Winder, Z. Liang, L. Antinori, P. J. Drew, N. Zhang, Time to wake up : Studying neurovascular coupling and brain-wide circuit function in the unanesthetized animal, NeuroImage 153 (Supplement C) (2017) $382-398$.

[2] A. K. O. Alstrup, D. F. Smith, Anaesthesia for positron emission tomography scanning of animal brains, Laboratory Animals 47 (1) (2013) 12-18.

[3] C. Carter, Brain Imaging in Behavioral Neuroscience, Vol. 11, Springer-Verlag Berlin Heidelberg, 2012.

[4] S. Cherry, Functional whole-brain imaging in behaving rodents, Nature Methods 8 (4) (2011) 301-303.

[5] V. D. Patel, D. E. Lee, D. L. Alexoff, S. L. Dewey, W. K. Schiffer, Imaging dopamine release with Positron Emission Tomography (PET) and 11C-raclopride in freely moving animals, NeuroImage 41 (3) (2008) 1051 - 1066.

[6] A. Z. Kyme, V. W. Zhou, S. R. Meikle, C. Baldock, R. R. Fulton, Optimised Motion Tracking for Positron Emission Tomography Studies of Brain Function in Awake Rats, PLOS ONE 6 (7) (2011) 1-16.

[7] D. Schulz, Integrating PET with behavioral neuroscience using RatCAP tomography, Reviews in the Neurosciences 22 (6) (2011) 647-655.

[8] J. Märk, D. Benoit, L. Balasse, M. Benoit, J. C. Clémens, S. Fieux, D. Fougeron, J. Graber-Bolis, B. Janvier, M. Jevaud, A. Genoux, P. Gisquet-Verrier, M. Menouni, F. Pain, L. Pinot, C. Tourvielle, L. Zimmer, C. Morel, P. Laniece, A wireless betamicroprobe based on pixelated silicon for in vivo brain studies in freely moving rats, Physics in Medicine \& Biology 58 (13) (2013) 4483.

[9] J. Godart, P. Weiss, B. Chantepie, J. C. Clemens, P. Delpierre, B. Dinkespiler, B. Janvier, M. Jevaud, S. Karkar, F. Lefebvre, R. Mastrippolito, M. Menouni, F. Pain, P. Pangaud, L. Pinot, C. Morel, P. Laniece, PIXSIC : A Pixellated Beta-Microprobe for Kinetic Measurements of Radiotracers on Awake and Freely Moving Small Animals, IEEE Transactions on Nuclear Science 57 (3) (2010) 998-1007.

[10] L. Balasse, J. Maerk, F. Pain, A. Genoux, S. Fieux, C. Morel, P. Gisquet-Verrier, L. Zimmer, P. Lanièce, PIXSIC, a Pixelated $\beta+$-Sensitive Probe for Radiopharmacological Investigations in Rat Brain : Binding Studies with [18F]MPPF, Molecular Imaging and Biology 17 (2) (2015) 163-167.

[11] L. Balasse, J. Maerk, F. Pain, A. Genoux, S. Fieux, F. Lefebvre, C. Morel, P. Gisquet-Verrier, P. Lanièce, L. Zimmer, PIXSIC : A Wireless Intracerebral Radiosensitive Probe in Freely Moving Rats, Molecular Imaging 14 (9).

[12] Ammour, et al., MAPSSIC, a Novel CMOS Intra-Cerebral $\beta+$ Probe for Deep Brain Imaging in Awake and Freely-Moving Rat : a Monte-Carlo Study, Submitted to IEEE Transactions on Radiation and Plasma Medical Sciences.

[13] J. Baudot, A. Besson, G. Claus, W. Dulinski, A. Dorokhov, M. Goffe, C. Hu-Guo, L. Molnar, X. Sanchez-Castro, S. Senyukov, M. Winter, Optimisation of CMOS pixel sensors for high performance vertexing and tracking, Nuclear Instruments and Methods in Physics Research Section A : Accelerators, Spectrometers, Detectors and Associated Equipment 732 (2013) 480 483.

[14] M. Mager, ALPIDE, the Monolithic Active Pixel Sensor for the ALICE ITS upgrade, Nuclear Instruments and Methods in Physics Research Section A : Accelerators, Spectrometers, Detectors 
and Associated Equipment 824 (Supplement C) (2016) 434 438. 